\title{
Vorteile der Verblisterung
}

\author{
Michael Felski
}

(C) Springer-Verlag Wien 2012

\section{Steigerung der Einnahmetreue durch verblisterte Medikamente}

In der Medizin spricht man von Compliance bzw. der Persistenz des Patienten als Oberbegriff für dessen kooperatives Verhalten im Rahmen der Therapie. Der Begriff kann mit "Therapietreue“" wiedergegeben werden. Durch die Verwendung von patientenindividuellen Blistern wird dem Pflegepersonal ein Hilfsmittel zur Hand gegeben, das es erlaubt, genau diese Einnahmetreue zu steigern und zu überwachen. Auf einen Blick ist erkennbar, welche Medikamente genommen worden sind und welche als nächstes genommen werden müssen. Ein Verwechseln von Einnahmezeitpunkten wird somit nahezu ausgeschlossen. Die Apotheke übernimmt die Verantwortung für die richtige Zusammenstellung der Medikamente und überprüft diese auf Wechselwirkungen und Kontraindikationen. Somit gewinnen alle Seiten: das Pflegepersonal gibt und überwacht die Einnahme der Medikamente, der Patient bekommt regelmäßig ,seine' Medikamente und der verordnende Arzt kann das erste Mal Rückschlüsse auf die Wirkung von verordneten Medikamenten und Therapien ziehen.

\section{Hygiene, Sicherheit und Verantwortung bei der Medikamentenversorgung}

Bei der Bereitstellung von Medikamenten im Pflegeheim kommt ein hohes Maß an Verantwortung auf das Pflegepersonal zu. Die Verwendung von verblisterten Medikamenten liefert hier Vorteile für alle Seiten. Der in der Apotheke

M. Felski $(\square)$

MTS Medication Technologies GmbH, Darmstadt, Deutschland gefüllte Kartenblister wird unter hygienisch einwandfreien, reproduzierbaren Bedingungen manipulationssicher hergestellt. Das Pflegepersonal wird somit zeitlich entlastet und kann sich bei der Gabe auf die von der betreuenden Apotheke bereitgestellten, verblisterten Medikamente eines jeden Zeitpunkts verlassen.

Sicherheit bezieht sich auf die Kontrolle der Medikamentenblätter des Patienten. Sicherheit bezieht sich auch auf die Reichweite von Medikamenten, die durch die Apotheke klar gewährleistet wird. Die Heimversorgungssoftware der Apotheken beinhaltet eine Reichweitenbestimmung, die es ermöglicht, dass Medikamente rechtzeitig per Rezept nachbestellt werden. Auch das Wissen um die Urlaubszeiten der Praxen ermöglicht eine bessere logistische Leitung des Medikamentenmanagements. Des weiteren gibt es durch die Entfernung der Medikamente aus dem Schwesternzimmer nicht mehr die Problematik der Fehlstellung von ,fremden Medikamenten. Die daraus resultierenden Einweisungen ins Krankenhaus werden minimiert. Für den verordnenden Arzt ergibt sich aus der Versorgung der Patienten mittels Blisterkarte auch, dass eine Medikamentenpackung mit 100 Tabletten wirklich für 100 Einnahmezeitpunkte reicht.

\section{Betreuung von arbeitenden Heimbewohnern}

Am Beispiel von Menschen mit Behinderung, z. B. Lebenshilfe, kann man sehr gut erkennen, wie eine sinnvolle Betreuung von Patienten mit verblisterten Medikamenten möglich ist. Für die normale Versorgung des Patienten zu den Zeiten, die er im Wohnbereich verbringt, wird eine Blisterkarte mit vier bis fünf Einnahmezeitpunkten verwendet. Da die Versorgung zur Mittagszeit bei der Arbeitsstelle auch gesichert sein sollte, und zwar in einem ähnlich sicheren und hygienischen System, wird von vielen Apotheken den Patienten 
ein zweiter Blister für die Mittagsmedikation bereitgestellt. Mit fünf Einnahmezeitpunkten bietet er die Möglichkeit, dass alle Medikamente der normalen Arbeitstage Montag bis Freitag gestellt werden können. Der Zusatzblister für die Mittagsmedikation wird an die Arbeitsstelle geliefert und vor Ort muss man sich dadurch nur noch um die Bereitstel- lung der Blister zum Mittagessen kümmern. Vorarbeiter und Meister werden somit aus der direkten Verantwortung für die Medikamentenbereitstellung genommen, was von allen Seiten als sehr positiv empfunden wurde. Die Selbständigkeit des Patienten bei der Medikamenteneinnahme zur Mittagszeit wird damit gefördert. 